\title{
Cochlear Schwannomas
}

\author{
Marco Barbieri, M.D., ${ }^{1}$ Michel Bruzzo, M.D., ${ }^{1}$ Renato Mora, M.D., ${ }^{1}$ \\ Renaud Meller, M.D., ${ }^{1}$ André Chays, M.D.,' and Jacques Magnan, M.D. ${ }^{1}$
}

\begin{abstract}
In a series of 179 cerebellopontine angle (CPA) tumors, the authors present nine cases $(5 \%)$ that were cochlear nerve neuromas. There were six men and three women (mean age, 51 years). Preoperative magnetic resonance imaging confirmed the diagnosis in one case with a labyrinthine extension and raised suspicions in the other four cases, which were confirmed during surgery. The remaining neuromas were discovered intraoperatively. The mean time between first observation and surgery was 9 months. Preoperatively, all patients underwent a complete otoneurological assessment. The middle fossa approach was used for the patient with the labyrinthine extension, and the retrosigmoid approach was used for the other eight cases. In all patients facial nerve function was preserved. Sudden or major hearing loss without associated vestibular symptoms or preoperative facial paralysis may be predictive of a cochlear component of a CPA tumor. The near-field relationships of cochlear neuromas located at the level of the acoustic and facial nerves can be appreciated because of their small size and strong contrast enhancement.
\end{abstract}

KEYWORDS: Cochlear neuroma, cochlear schwannoma

\begin{abstract}
Acoustic nerve neuromas or schwannomas most frequently develop from the inferior vestibular nerve sheath. ${ }^{1-3}$ Benign tumors, they progressively develop in and outside the internal auditory canal (IAC). These tumors were first described by Sandiford de Leyden in 1777 from an autopsy. In 1830 Bell reported their clinical features, followed by Schlitter in 1930 (quoted in Charachon et al. ${ }^{1}$ ) and Fowler in $1936 .{ }^{4}$ With the diagnostic advances offered by magnetic resonance imaging (MRI), neuromas are now diagnosed early in their course, mak-
\end{abstract}

ing it possible to detect the nerve of origin both by imaging as Leonetti describes ${ }^{5}$ or intraoperatively. We reported nine such cases treated in 5 years.

\section{MATERIALS AND METHODS}

From October 1993 to December 1998, 178 patients with 179 tumors of the cerebellopontine angle (CPA) underwent surgery in our ENT

Skull Base, volume 11, number 4, 2001. Address for correspondence and reprint requests: Jacques Magnan M.D., ORL Unit, Hôpital Nord, 13915 Marseille Cedex 20, France. 'ORL Unit, Hôpital Nord, Marseille, France. Copyright @ 2001 by Thieme Medical Publishers, Inc., 333 Seventh Avenue, New York, NY 10001, USA. Tel: +1(212) 584-4662. 1531-5010,p;2001,11,04,241,244,ftx,en; sbs00236x. 
department. Of these 179 tumors, 130 were resected through the retrosigmoid approach (tumors smaller than $2.5 \mathrm{~cm}), 119$ with the goal of preserving hearing (49\% preserved, $51 \%$ lost); 39 through the translabyrinthine approach, 4 through the preand retrosigmoid approach for Stages IV and V, and one through the middle fossa approach.

Of the 179 acoustic neuromas considered, nine showed evidence of arising from the cochlear segment of the VIIIth cranial nerve. The nine patients (six men, three women; mean age, 51 years) with a cochlear neuroma were studied to determine the clinical, audiological, and radiological features of this neglected group of tumors. The mean time between first observation and surgery was 9 months. All patients were monitored with a full otoneurologic test, and audiovestibular balance was evaluated. Tonal audiometry identified the average parameters of bone conduction, and vocal testing defined the discrimination speech score. For the nine patients, audiometry showed a mean neurosensorial hearing loss of $64.8 \mathrm{~dB}$. Hearing thresholds ranged between 40 and $96 \mathrm{~dB}$. Five patients also had tinnitus. The hearing of four patients rapidly deteriorated within 3 to 6 months. None of the patients, however, had vestibular symptoms. One patient had homolateral facial paralysis that recovered a few days before surgery.

Axial and coronal T1- and T2-weighted MRI with gadolinium enhancement identified the size and location of the tumor and in four cases raised the suspicion of cochlear neuroma as the diagnosis. Tumor extension at the cochlear level confirmed the diagnosis in one case. Four tumors were $6 \mathrm{~mm}$, two were $5 \mathrm{~mm}$ (Fig. 1), one was $7 \mathrm{~mm}$, and two were $8 \mathrm{~mm}$. According to Zini-Magnan classification $^{6}$ (Table 1 ), five patients were $\mathrm{Ib}$, one patient was IIb, and three were Ic. The auditory brainstem response showed an abnormal increase in latency $\mathrm{I}-\mathrm{V}$ in four cases and a desynchronization in one case. Eight patients underwent a retrosigmoid approach, and one underwent a middle fossa approach. Postoperatively, facial nerve function was monitored, and a control audiogram was

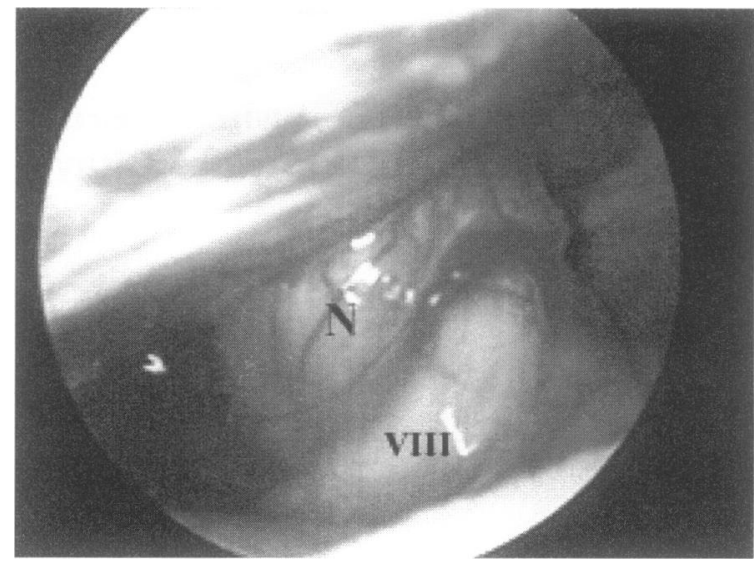

Figure 1 Left-sided acoustic neuroma with a 5-mm extension into the cerebellopontine angle. The acoustic neuroma is below the auditory nerve and not between the cochlear and vestibular segments as usual. $N$, neuroma; VIII, 8th cranial nerve.

obtained. All operative specimens were sent for a histopathological examination.

\section{RESULTS}

The retrosigmoid approach allowed the IAC to be opened and the entire tumor to be visualized. Adherence of the neuroma to the cochlear nerve was

Table 1 Zini-Magnan Tumor Classification6

\begin{tabular}{ll}
\hline Stage $^{\dagger}$ & Features \\
\hline 1 & Intracanalicular tumor \\
2 & Small tumor, up to $10 \mathrm{~mm}$, in CPA, no contact \\
& with brain stem \\
3 & Middle-size tumor, 11 to $20 \mathrm{~mm}$, within CPA, \\
touches and embedded in brain stem without \\
distortion
\end{tabular}

CPA, cerebellopontine angle

${ }^{+}$For each stage we used a further classification according to intracanalicular extension:

A: Internal auditory canal (IAC), tumor process Absent

B: IAC Bottom free of tumor process

C: IAC Completely filled 
confirmed during intracapsular dissection of the tumor from the nervous component of the acousticofacial bundle and confirmed endoscopically. The vestibular nerve was intact, and the tumor was connected to the medial end of the cochlear nerve. In all cases the course of the vestibular nerve was visible and the nerve was sectioned at the end of the dissection to avoid the onset of a disabling postoperative syndrome caused by the lack of vestibular compensation. The patient with the intracochlear neuroma underwent the middle fossa approach to determine if the tumor had a labyrinthine extension. The diagnosis of schwannoma was always confirmed by the pathologist. The tumors were on the left side in eight patients and on the right in one patient. Facial nerve function was preserved in all cases, but all patients were deaf, as expected.

\section{DIscussion}

The first surgical excision of a neuroma dates to Balance in 1894. ${ }^{1}$ Later Henschen ${ }^{7}$ in 1915 and Skinner in $1929^{8}$ reported that these tumors originated from the vestibular nerve. They suggested that abnormal placement of the vestibular nerve in its sheath may be predictive of a schwannoma. Since then, acoustic neuromas have been considered exclusively to be vestibular schwannomas associated with unilateral progressive hearing loss as their major symptom.

In our study, the most frequent presenting symptom was a major neurosensorial hearing loss, which was unrelated to the size of the tumor. Four patients (45\%) experienced an abrupt decrease in hearing function within 3 to 6 months. Such rapid deterioration was reported by Chays et al in $1996^{2}$ and Leonetti in $1998 . .^{5}$ In our study speech discrimination followed the same pattern as reported by Leonetti. ${ }^{5}$

Tinnitus occurred in five of our patients; Leonetti ${ }^{5}$ reported that only two of 16 patients with cochlear schwannoma had tinnitus. In their study of postmortem cases, Ayani ${ }^{10}$ and Johnsson ${ }^{11}$ reported no tinnitus in previous anamnestic data.

Some authors ${ }^{1,2,8,9}$ have correlated the presence of a vertigo syndrome to the evolution of cochlear neuromas. Thomsen and Jorgensen ${ }^{12}$ compared three cochlear nerve neuromas to autopsies in asymptomatic patients; they concluded that, for that diagnosis, a very small tumor was required to locate its origin accurately in the IAC. This conclusion agrees with our data: tumor size ranged between 5 and $8 \mathrm{~mm}$.

A particular feature, that is, a reversible facial paralysis on side of the tumor, was noticed in one of our patients. The literature does not mention facial paralysis although the facial nerve has been found to be compressed in cadaveric studies ${ }^{8}$ with no clinical sign of damage to facial nerve function.

MRI is the key test for accurately diagnosing this disorder and for determining the location of tumors. MRI always detects neuromas, even when they are very small. Tumors as small as $1 \mathrm{~mm}$ can be evaluated and graded. Leonetti ${ }^{5}$ suggested that MRI is the most reliable diagnostic tool, showing cochlear tumors in the inferior compartment of the anterior wall of the IAC. This finding was recently confirmed by Krueger and Kemper. ${ }^{9}$ Assessment with MRI allowed us to suspect the diagnosis of cochlear neuroma diagnosis in four patients and to be certain in one case.

Postmortem cases of an intracochlear or intracanalicular neuroma (one case in our study) or both have been described ${ }^{10,11,12}$ in patients who had no hearing problems while alive. Our patient noted an abrupt but reversible hypoacusia and incapacitating tinnitus followed by a new and sudden irreversible hearing loss that resolved into profound deafness 6 months later.

The minimally invasive retrosigmoid approach, performed in eight patients, is indicated for small tumors (less than $2 \mathrm{~cm}$ ) of the CPA and to preserve hearing. ${ }^{13,14}$ This approach has greatly benefited from endoscopic techniques ${ }^{15-18}$ which accurately delineate the relationship between the tumor and its origin (Fig. 2). "En bloc" dissection 


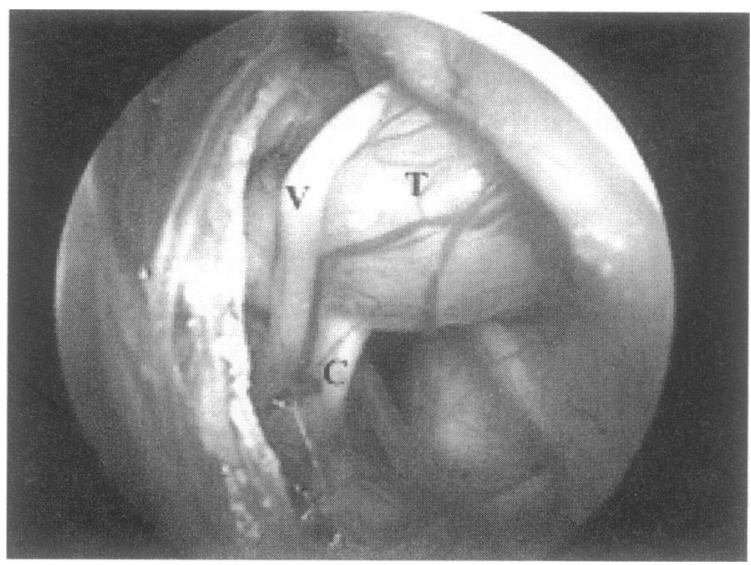

Figure 2 Right-sided acoustic neuroma from the cochlear nerve. The vestibular nerve is intact along its course, and the neuroma is located posteroinferiorly. $\mathrm{V}$, vestibular nerve; $C$, cochlear nerve; $T$, tumor.

of a small cochlear neuroma is a safe procedure in terms of the facial nerve whereas the vestibular nerve delineates a helpful cleavage plane between the vestibulofacial nerve bundle and the tumor adherent to the cochlear nerve.

\section{CONCLUSION}

Although more rare than vestibular nerve schwannomas, cochlear nerve schwannomas are not exceptional, as indicated by their $5 \%$ rate of occurrence in our series. Radiological data provided by MRI of a small acoustic nerve tumor associated with rapidly progressive hypoacusia and the absence of vertigo are irrefutably predictive of a cochlear nerve tumor. MRI also can show if a neuroma is located along the cochlear nerve or if it extends into the cochlea.

\section{REFERENCES}

1. Charachon R, Lavielle JP, Chirossel JP. Neurinome de l'acoustique. Encycl Med Chir (Elsevier Paris) ORL; 1999:2, 20250 A-10

2. Chays A, Contant A, Locatelli P, Caces F, Lebreuil G, Magnan J. Neurinome du nerf cochleaire. Ann Otolaryngol Chir Cervicofac 1996;113:339-347

3. Tos M, Thomsen J. Acoustic Neuroma. Amsterdam: Kugler Publications; 1992

4. Fowler EP. Acoustic tumors within the internal auditory meatus. Laryngoscope 1936;46:616-627

5. Leonetti JP. Cochlear neuromas. Am J Otol 1998;19: 499-502

6. Zini C, Magnan J, Piazza F, Chays A, Passanisi E, Girard $\mathrm{N}$. New radiosurgical classification of acoustic neuromas. In: Sanna M ed. Acoustic Neurinomas and Other CPA Tumors. Roma: Monduzzi edit; 2000:117-120

7. Henschen F. Zur histologie un pathogenese der kleinhirnbruckenwinkel-tumoren. Arch Psychiatr Nervenkr 1915; 56:121-122

8. Skinner HA. Origin of acoustic nerve tumours. Br J Surg 1929;16:440-463

9. Krueger W, Kemper V. Cochlear schwannomas. Skull Base Surg 2000;10:87-88

10. Ayani N, Shea JJ. Asymptomatic schwannoma of the cochlear nerve. Arch Otolaryngol 1982;108:501-503

11. Johnsson LG, Kingsley TC. Asymptomatic intracochlear neurinoma. Arch Otolaryngol 1981;107:377-381

12. Thomsen J, Jorgensen MB. Undiagnosed acoustic neuroma. Arch Otolaryngol 1973;204:175-182

13. Bremond GA, Garcin M, Magnan J. Progres en otoneurochirurgie: l'abord a minima de l'angle ponto-cerebelleux par voie retrosinusale. Acta Otolaryngol Belg 1976;301:127-144

14. Shelton C, Alavi S, Li JC, Hitselberger WE. Modified retrosigmoid approach used for selected acoustic tumor removal. Am J Otol 1995;16:664-668

15. Magnan J, Chays A. Voie retro sigmoide et endoscopie de l'angle ponto-cerebelleux: indications et resultats. J Fr ORL 1994;43:347-352

16. O'Donoghue GM, Greengrass ST, Magnan J. Endoscopy in otology and otoneurosurgery. Adv Otolaryngol Head Neck Surg 1994;8:43-67

17. Magnan J, Sanna M. Endoscopy in neuro-otology. Stuttgart: Georg Thieme Verlag; 1999

18. Bruzzo M, Broder L, Duflo S, Chays A, Magnan J. Cochlear neuromas, fact or fantasy? In: Sanna $M$ ed. Acoustic Neurinoma and Other CPA Tumors. Roma: Monduzzi edit; 2000:217-223 